\title{
Orientación de los significados emocionales de profesoras durante su trabajo en aula [Orientation of the emotional meanings of
} teachers during their work in the classroom] Octavio Poblete-Christie (1) Universidad de Playa Ancha

\begin{abstract}
Resumen
Este artículo expone parte de los resultados de una investigación cuyo objetivo fue explorar diversos aspectos de la experiencia emocional de profesoras en aula. Específicamente, estos resultados se obtuvieron fruto del análisis de los significados construidos sobre dicha vivencia. Se utilizó un enfoque cualitativo y un diseño en base a episodios emocionales, es decir, situaciones concretas de clases en las cuales surgieron emociones en las docentes. Participaron cuatro profesoras de enseñanza básica de las asignaturas de lenguaje y matemáticas de dos establecimientos diferentes. Como técnicas de construcción de datos se recurrió a entrevistas semiestructuradas. Se estudiaron 20 episodios, realizándose 20 entrevistas, una por cada episodio. El análisis de contenido mostró diversos significados que explicaron el surgimiento de las emociones experimentadas a partir de los cuales fue posible construir las categorías de orientación externa, internay mixta. Se discute su implicancia en base a criterios psicológicos, educativos y epistemológicos.
\end{abstract}

Palabras clave: emociones, profesoras, significados, afecto, episodios emocionales.

\begin{abstract}
This article presents some of the results of research into various aspects of the emotional experience of classroom teachers. Specifically, the results were obtained through analysis of the meanings constructed in relation to their work. We used a qualitative approach and a design based on emotional episodes, that is, concrete classroom situations that provoked emotions in teachers. Four primary school mathematics and Spanish language teachers from two institutions participated. We used semi-structured interviews as data construction techniques. We studied twenty episodes, conducting twenty interviews, one for each episode. The content analysis yielded various meanings that explained the emergence of the emotions experienced, from which we were able to construct three categories: external, internal, and mixed. We discuss the implications based on psychological, educational, and epistemological criteria.
\end{abstract}

Keywords: emotions, teachers, meanings, affection, emotional episodes. 


\section{INTRODUCCIÓN}

Durante las últimas décadas los afectos han acaparado la atención de los investigadores, trayendo a la palestra una dimensión de lo mental cuyo valor para la comprensión del comportamiento humano es reconocido cada vez con más fuerza. Esta labor ha sido nutrida desde un amplio abanico de disciplinas como la filosofía (e.g., Nussbaum, 2008), la neurociencia (e.g., Le Doux, 1998), las ciencias sociales (e.g., Lara \& Enciso, 2013), entre muchas otras. Asimismo, el feminismo ha relevado el valor de esta dimensión que históricamente ha sido ignorada por el modernismo (Solana \& Vacarezza, 2020). Es que se trata de un objeto de estudio especialmente complejo caracterizado por tensionar las concepciones epistémicas de nuestra tradición cultural (Damasio, 1999).

En este contexto, debido muy posiblemente a que constituyen la manifestación más saliente de la vida afectiva y por tener un influjo significativo sobre la interacción humana, las emociones han concitado un interés especial. Sin embargo, pese a su naturaleza tangible que las distingue de otros fenómenos de interés psicológico y social, como por ejemplo la autoestima o las actitudes, en el esfuerzo por comprenderlas se contraponen diversas teorías y epistemologías. Asimismo, dichas conceptualizaciones suelen reñirse entre sí en función distintas dicotomías que han acompañado el pensamiento moderno tales como cuerpo/mente, razón/emoción, privado/público y naturaleza/cultura. Esta última resulta especialmente relevante para este trabajo.

\section{Del innatismo a la cultura}

La confrontación entre posiciones innatistas y culturalistas ha caracterizado el esfuerzo por comprender las emociones, siendo Darwin (1872) el primero que abordó su estudio científico desde una posición evolutiva. Posteriormente, en 1884 destaca un breve ensayo de Willams James sobre el efecto de la activación fisiológica sobre la experiencia emocional (Aguado, 2005). Mucho después, desde la década de los 70 del siglo pasado, el interés por las emociones reapareció en torno su expresión y reconocimiento (Ekman \& Oster, 1979). A partir de esta idea, se ha desarrollado una línea de investigación centrada en lo que se denominó como emociones básicas cuya influencia se extiende hasta nuestros días. Sin embargo, otros autores han argumentado el carácter social, cultural, e histórico de estos estados mentales. Por ejemplo, Bruner (1986) postuló que diferentes contextos culturales construyen realidades emocionales distintas y que los estados emocionales de cada persona aparecen en función de los contextos comunicativos establecidos durante su historia, principalmente fruto del contacto temprano con sus cuidadores. Es así como el ambiente influiría de manera determinante sobre la manera de interpretar emocionalmente la vida, configurando una suerte de "rúbrica" para tales fines.

Congruente con aproximaciones de este tipo se ha generado una gran cantidad de evidencia empírica 
que muestra cómo al interior de contextos culturales distintos surgen modos diferentes de significar, percibir, y sentir las emociones (pe. Boiger et al., 2018, Gendron, et al., 2014; Lim, 2016; Mascolo et al., 2003; Rodríguez, 2015; Romero et al., 2015). Asimismo, en esta categoría es posible incluir los trabajos realizados desde la psicología social crítica que muestran cómo las emociones son hechos construidos en la interacción, participando factores individuales, sociales y procesos de mediación que funcionarían articulando los dos primeros (Belli \& Íñiguez-Rueda, 2008). En esta misma línea de análisis cabe destacar el trabajo desarrollado desde los años setenta del siglo pasado por la sociología de las emociones (Bericat, 2000). De particular interés para este escrito son los aportes de Hochschild (1975) quien puso de manifiesto que las emociones están impregnadas de significados propios del contexto sociohistórico, destacando como consecuencia de lo anterior la presencia en la sociedad de normas emocionales (feeling rules). Asimismo, de manera particular, documentó el control que ejercen determinadas empresas sobre las emociones de sus trabajadores debido a criterios eminentemente comerciales, explicitando lo que denominó como labor emocional (Hochschild, 2008). Por otra parte, Theodore Kemper (1978) teorizó sobre el surgimiento de las emociones en espacios relacionales, recurriendo para ello a diversos procesos de reforzamiento que se desplegarían en función del poder y estatus percibido. En este sentido, distinguió entre emociones de valencia positiva que surgirían en un contexto de equilibrio y otras de valencia negativa que surgirían en un contexto de desequilibrio (e.g., culpa, vergüenza, miedo-ansiedad, depresión), es decir, fruto de un exceso o déficit percibido de dichas dimensiones. Como se puede ver, existe bastante literatura que ha ido argumentando el origen social de las emociones. Justamente, considerando esta aparente oposición entre naturaleza y cultura, es que resulta interesante revisar una de las teorías neurocientíficas más recientes sobre este tema.

\section{Emociones ¿Hechos objetivos o construidos subjetivamente?}

En el último tiempo se ha cuestionado la posibilidad de que las emociones sean hechos objetivos, innatos, y discretos, apuntando a que más bien correspondan a construcciones psicológicas y sociales (Barrett, 2018; Barret \& Russell, 2015). Barrett (2018) ha obtenido una evidencia importante que apoya la idea de que no existen huellas o marcadores físicos (objetivos) que permitan a cualquier observador identificar emociones, ya sea propias o de otra persona. Estos estudios han mostrado que no existe homogeneidad en el surgimiento de las emociones, sino total variabilidad. Para entender cómo el cerebro construye una emoción es necesario considerar que éste recurre a un sistema de conceptualización que otorga coherencia a la información proveniente desde los órganos sensoriales por medio de conceptos desarrollados fruto de la interacción con el medio; y a su vez, recurre a señales interoceptivas que se activan fruto del movimiento de nuestro cuerpo y que, como resumen, arrojan en cada momento sensaciones que se pueden describir en base a las dimensiones de valencia e intensidad (afecto 
nuclear) (Barrett, 2018; Barrett \& Russell, 2015). Así, con base en estos dos procesos, cada persona construye lo que para sí misma es una emoción. Finalmente, la actividad interoceptiva influye en cómo percibimos el mundo. De esta forma, podemos percibir un parque como un lugar relajante o como un lugar extremadamente amenazante dependiendo de nuestra experiencia vivida en este tipo de lugares.

\section{Significados y emociones}

A diferencia del intento por entender la mente realizado por el cognitivismo (Varela et al., 1997), los significados permiten tener una visión más integrada del comportamiento humano al articular la vida de las personas con su contexto socio cultural. Por ello los significados han pasado a constituirse en un aspecto central en el estudio de la cognición (Pozo, 2006). Fruto del intercambio con la comunidad a la que se pertenecen, las personas elaboran versiones normativas que les permiten entender el mundo y la realidad cultural en que viven. A su vez, estos significados son constantemente revisados y reconstruidos a través de un proceso cotidiano de negociación. Es decir, los significados permiten que las personas entiendan la realidad en que viven, los que a su vez son usados como herramientas que permiten transformar dicha realidad (Bruner, 1991).

Desde la teoría de la emoción construida, el afecto nuclear está a la base de la generación de los significados. Éste provoca que con apoyo de la conceptualización que nos proporciona nuestra cultura interpretemos una determinada situación de una forma u otra. Producto de la experiencia, el cerebro maneja un modelo sobre cómo será el mundo en el siguiente instante, creando significados a partir del mundo y de nuestro cuerpo, y para lo cual usa conceptos, creación que responde a nuestro contexto sociocultural.

\section{El estudio de las emociones en educación}

Aunque el interés por las emociones surge desde diferentes ámbitos de la actividad humana, en el sistema educativo han ido ocupando un lugar protagónico, algo que se explicaría principalmente por tres razones. La primera tiene que ver con el influjo que ejercen estos afectos sobre los procesos de enseñar y aprender (Pekrun \& LinnenbrikGarcia, 2014). La segunda de ellas tiene que ver con la conexión entre estos estados mentales, el trabajo docente y la salud mental de las y los profesores y -con una contribución importante desde la sociología- entre esta última y las políticas públicas (Tsang, 2014). Y una tercera razón, íntimamente relacionada con las dos anteriores, apunta al tipo de conocimiento que se requiere para poder utilizar las emociones a favor del aprendizaje de los contenidos escolares, del desarrollo socioemocional de las y los estudiantes, y del bienestar subjetivo de las y los profesores. Esto último constituye un motivo de mayor alcance pues interpela de manera simultánea nuestras concepciones culturales sobre lo que consiste conocer y educar. De esta forma, dado que la escuela ha sido diseñada para transmitir el acervo de conocimientos a las nuevas generaciones, la asimilación de la vida afectiva como parte central del conocer constituye un desafío cuyos efectos transformadores no es difícil imaginar, no solo sobre la institucionalidad educativa en 
particular sino sobre la sociedad en general (Naranjo, 2007). Es así como, la investigación sobre estos afectos en estos actores se ha transformado en un ámbito de interés cada vez más creciente.

\section{La investigación sobre emociones en profesores}

El estudio de las emociones de las y los docentes ha concitado de manera persistente el interés de los investigadores desde fines del siglo XX (e.g., Chen et al., 2020; Keller et al., 2014; Saric, 2015; Uitto et al., 2015; Xu, 2018; Zembylas \& Schutz, 2016). La complejidad de este objeto de estudio puede observarse en la diversidad de aristas investigativas que desde éste se han desarrollado. Se ha indagado sobre aspectos como como la identidad del profesor y su formación profesional; el agotamiento emocional de los profesores y su conexión con el burnout y bienestar; las relaciones con estudiantes y otros actores educativos; la influencia de los contextos sociales, históricos y políticos; la inteligencia emocional y los procesos de regulación emocional de los profesores (Uitto et al., 2015). Asimismo, se ha explorado sobre el papel que cumplen las emociones de estos actores sobre diversos aspectos de los procesos educativos (e.g., Arias \& Rodríguez, 2019; Brackett et al., 2013; Chen et al., 2020; De Sixte \& Sánchez, 2012;
Funes, 2016; Hyry-Beihammer et al., 2019; Khajavy et al., 2018; Rodríguez-Corrales et al., 2017; Timoštšuk et al., 2016; Zenteno-Osorio \& Leal-Soto, 2016). A su vez, se ha trabajado en función de diferentes conceptualizaciones, recurriendo a aproximaciones cognitivas (e.g., Pekrun et al., 2007) y culturalistas (e.g., Hargreaves, 1998; Zembylas, 2002).

Sin embargo, la mayor parte de los estudios sobre esta materia han sido objeto de diferentes cuestionamientos tales como su desconexión con la investigación sobre estos estados mentales (Saric, 2015), la falta de claridad conceptual que muestran la mayor parte de los trabajos realizados (Fried et al., 2015), o el uso exclusivo de palabras emocionales para describir estos estados mentales (Saric, 2015). En este sentido, pese al avance logrado, este campo de indagación puede considerarse en un nivel exploratorio de desarrollo. Por ello, este estudio tuvo como objetivo explorar la experiencia emocional de profesores en aula, es decir, la vivencia consciente de sus emociones (Rosselló \& Revert, 2008), indagando sobre diversos aspectos tales como sus aspectos fenomenológicos y corporales, significados, factores biográficos de las participantes, y factores culturales que incidieron en cada episodio. En este escrito se presenta algunos resultados sobre los significados relacionados con la experiencia emocional de los docentes.

\section{MÉTODO}

Tomando como referencia la teoría de la emoción construida (Barrett, 2018), se realizó una investigación cualitativa, utilizando un diseño fenomenológico en base a episodios emocionales, es decir, situaciones concretas en que docentes experimentaron alguna emoción en aula. La investigación se realizó entre mayo y octubre de 2018, en la Región Metropolitana, Chile. 
Considerando el predominio de mujeres en la actividad docente, participaron cuatro profesoras en el estudio. Como criterio de selección se consideró su nivel de experiencia, (cinco o más años de trabajo en aula) y las asignaturas que impartían, esto es, lenguaje y matemática, dada su importancia para el sistema escolar. Se trabajó con profesoras de ambas asignaturas en los dos establecimientos diferentes. El estudio fue llevado a cabo en el segundo ciclo básico, tomando en cuenta algunos datos que apuntan a que en este nivel se observarían comportamientos más disruptivos (Kadesjö et al., 2004; Matson et al., 1983) provocando, por tanto, con más facilidad emociones intensas en sus profesores.

A fin dar respuesta a las objeciones al auto reporte como única estrategia para la construcción de datos (Frenzel, 2014; Phelps \& Sharot, 2008), el investigador participó en la selección de los episodios, observando directamente el comportamiento de las profesoras en clases e infiriendo a partir de su lenguaje no verbal el surgimiento de emociones en cada una de ellas. Se utilizó un sistema especialmente diseñado para observar y registrar por escrito el comportamiento de las profesoras de tal manera que, posteriormente, dicha información pudiera cotejarse fácilmente con los reportes que ellas mismas hicieran de sus emociones. Se seleccionaron 20 episodios emocionales, cinco por cada profesora. Previo al proceso de observación se incluyó un periodo destinado a que las docentes y sus estudiantes se habituaran a la presencia del investigador en aula. Al finalizar cada clase, cada profesora comparó sus impresiones con lo registrado por escrito por el investigador y cuando ambos coincidieron en la presencia de un episodio emocional, éste fue seleccionado. En ese mismo momento y para evitar los efectos del olvido, la profesora reportó brevemente los aspectos centrales del episodio y de lo que experimentó durante éste.

Posteriormente se realizó una entrevista semiestructurada a fin de profundizar sobre lo ocurrido. El diseño de dicha entrevista consideró aspectos de la entrevista episódica (Flick, 2012) y de la entrevista microfenomenológica (Petitmengin, 2017). En estos encuentros las profesoras relataron los acontecimientos, describieron las emociones experimentadas y reflexionaron de manera amplia sobre diversos aspectos relacionados con lo ocurrido. En total se realizaron 20 entrevistas (una por cada episodio). Tanto los reportes realizados al finalizar cada clase como las entrevistas fueron registrados en audio $\mathrm{y}$ posteriormente transcritos. Previo a la realización del estudio tanto las autoridades y directores de cada establecimiento como las profesoras participantes firmaron un formulario de consentimiento informado a través del cual se les explicó los objetivos, metodología y confidencialidad de los datos, clarificando el carácter voluntario de su participación.

Los datos fueron analizados usando análisis cualitativo de contenido (Cáceres, 2003) de las referencias de las profesoras sobre los significados relacionados con las emociones experimentadas. El análisis, de carácter inductivo, se efectuó con ayuda del programa ATLAS.ti versión 7.2.18. El procedimiento de análisis sobre los significados fue similar al realizado junto a otros investigadores y estuvo focalizado en las explicaciones que las profesoras dieron sobre el surgimiento de sus 
emociones. Cabe destacar que, además de su carácter interoceptivo, las emociones tienen un origen interpretativo, lo cual ha dado pie a plantear que éstas son en sí mismas significados (Barrett, 2018). Visto de esta forma, asumimos que las explicaciones analizadas tendrían un valor ontológico pues surgirían de interpretaciones espontáneas que dispararon las emociones sentidas por las profesoras. Debido a lo anterior hemos denominado a estas explicaciones como significados nucleares y fueron codificadas a través de expresiones que preservaron lo mejor posible los términos utilizados originalmente por las profesoras, obteniendo una serie de códigos en vivo (Albornoz et al., 2015). El siguiente ejemplo muestra la fundamentación de uno de los códigos en vivo, en el cual la profesora explica su molestia debido a la poca tolerancia que observó en sus estudiantes por criticar el video musical que ella utilizó en su clase, sin considerar que el objetivo de exponerlo era ejemplificar los distintos tipos de amor y no evaluar preferencias musicales personales:
Código: Me molestó que hayan sido poco tolerantes [...].

Profesora: Es porque siento que no fueron tolerantes al ver que en un contexto educativo las canciones venían de distintas temáticas y distintas épocas, porque había desde rock, desde punk, hasta reguetón, entonces cualquier otro que no le guste el punk, puede haberme dicho o cualquier otro que no le gusta la música romántica, me pudo haber dicho. Eso es lo que yo siento, que no se contextualizaron en la clase, sino que vieron la tendencia musical no más, siendo que el foco era buscar la experiencia del amor y no ver los gustos musicales de cada uno (Episodio 10).

En varios episodios las profesoras señalaron más de un factor para explicar su emoción siendo posible identificar en esos casos más de un significado nuclear. Con base a los significados nucleares fue posible construir, también de manera inductiva, tres categorías. A fin de enriquecer la riqueza interpretativa y analítica, al proceso de análisis fue sometido a un proceso de triangulación con otros investigadores (Hernández et al., 2014).

\section{RESULTADOS}

A continuación, se exponen y fundamentan las categorías orientación interna, externa, y mixta construidas a partir de los significados nucleares.

\section{Orientación interna}

Esta categoría envuelve aquellas emociones cuyos significados nucleares se refirieron principalmente al comportamiento o la actividad mental de la propia profesora. Un ejemplo de esta categoría se relaciona con un cambio notable de disposición en uno de los estudiantes provocando felicidad en la profesora. Dicho cambio fue interpretado como producto del trabajo que ella había realizado con ese estudiante durante el año. Es decir, la emoción fue explicada por algo interno, un proceso formativo que ella había realizado con el estudiante.

Profesora: Me siento feliz por haber logrado cosas con él.

Entrevistador: A ver cuéntame un poquito eso. 
Profesora: Porque como decíamos, [él] tenía otra actitud antes, aunque igual pasa por sus momentos, entonces claro, versus a lo anterior y ahora, está en un camino muy grande ha recorrido, casi de extremo a extremo, entonces obviamente eso genera que uno sienta que ha logrado cosas con él, que dentro de todas las cosas que uno le puede decir, que muchas veces dice que no le importa, algo le llegó, algo pasó por su cabeza que está haciendo que tenga una actitud diferente (Episodio 14).

Una situación análoga, aunque de valencia opuesta, ocurre cuando otra profesora experimenta rabia a partir de las interrupciones de los estudiantes. Sin embargo, ella interpreta dicha emoción en función de ciertos rasgos personales.

Profesora: Yo soy muy estructurada en tanto lo que es una clase y que un ambiente para poder trabajar tiene que ser ojalá en silencio, trabajando cada uno, consultando sus dudas, entonces (...) unos se desconcentran producto de él, porque les empieza a hablar, porque el garabato, que aquí, allá, entonces eso para mí me genera rabia porque no puedo hacer las cosas como me gustaría que fueran (Episodio 11).

\section{Orientación externa}

Esta categoría envuelve aquellas emociones cuyo significado nuclear estuvo referido principalmente al comportamiento de los estudiantes, es decir, algo externo a la profesora. Un caso que ejemplifica esta situación es la desilusión experimentada por una de las docentes debido a que los estudiantes no lograron los aprendizajes que ella y la profesora de apoyo habían intentado propiciar en dicha ocasión. Además, en dicha clase habían asistido pocos estudiantes, favoreciendo un trabajo más focalizado y cercano, todo lo cual debiera haber generado mejores resultados.

Código: Me provocó desilusión que los estudiantes aprendieran muy poco [...]

Profesora: Creo haber logrado que los ocho alumnos presentes, entendieran lo que se trataba de lograr, el objetivo propuesto, pero en el momento en que con la profesora del PIE empezamos a hacer preguntas, nos dimos cuenta o me doy cuenta de que los alumnos aprenden muy poco y eso me provoca desilusión (Episodio 1).

De manera similar, otra profesora explicó su molestia a partir del desinterés de los estudiantes a utilizar los recursos que ella les había ofrecido sistemáticamente durante la clase para que pudieran realizar una tarea. Al ser consultada por la razón de su molestia en ese momento, dijo: "que no hayan leído el libro, fue que no ocuparon las herramientas que yo les facilité" (Episodio 18).

\section{Orientación mixta}

Esta categoría envuelve aquellas emociones sobre las cuales se construyeron significados nucleares referidos tanto al comportamiento o la actividad mental de la propia profesora como al comportamiento o actividad mental de los estudiantes. Es decir, la emoción fue explicada por algo que estaba ocurriendo simultáneamente fuera y dentro. Un ejemplo de esto ocurrió cuando un estudiante informó abiertamente durante la clase que no iba a asistir a un paseo educativo que estaba programado. Ante esta 
situación, la profesora experimentó angustia por no saber las razones por las cuales el estudiante no deseaba asistir al paseo, considerando que, pese a que el estudiante era nuevo en el grupo, había logrado integrarse muy bien con sus compañeros.

Código: Me angustió no saber por qué no quería ir [...].

Profesora: Que de repente el niño dijera y si yo no quiero ir, y ¿por qué no quiere ir? 'No, no quiero ir' y es como ¿por qué no quiere ir ahora si antes quería ir? (...) No me calza, dije ¿qué pasó aquí?, por qué no quiere ir, por qué no quiere participar si yo lo he visto integrado con los compañeros desde que llegó, hay niños que llegan a un curso a mitad de año, a principio de año y cuando el curso está conformado del año anterior les cuesta más adaptarse, pero él inmediatamente se sentó con un compañero, conversa con los otros, y a mí me sorprendió, ¿por qué no quiere ir ahora?, ¿pasó algo en este momento? (Episodio 9).

Por otra parte, esa misma emoción fue explicada por la profesora por algo "externo". La posibilidad de que le hubiera ocurrido algún hecho negativo al estudiante que no quería ir al paseo es señalada como motivo de la angustia. Y dicha eventualidad se explicaría, según la profesora, por la existencia un posible conflicto entre dicho estudiante y otro miembro del curso que le hubiera generado molestia, provocando en definitiva su negativa a asistir al paseo.

Código: Me angustió que pudiera estar pasando algo malo que yo no sabía [...]

Entrevistador: ¿Tú me quieres decir con eso que la angustia tiene que ver con que pudo haber pasado algo a él?

Profesora: A él, con el curso, peleó con alguien. Entrevistador: ¿Ocurrió algo?

Profesora: Ocurrió algo en el curso que yo no sé. Claro, que él no quiere compartir con los otros, eso yo pensé altiro, por eso me angustié. Entrevistador: Como que ocurrió algo mal con él o con el curso.

Profesora: Con el curso, él está molesto con alguien del curso (Episodio 9).

\section{CONCLUSIONES}

La totalidad de los significados nucleares construidos responden a parámetros propios del sistema escolar (autores, en revisión), es decir, son congruentes con un determinado contexto sociocultural (Barrett, 2018; Bruner, 1998; Hochschild, 1975). Por tanto, los datos obtenidos permiten mostrar cómo la institucionalidad educativa "construye" la experiencia emocional de las profesoras (Fried et al., 2015; Hargreaves, 1989; Tsang, 2014; Zembylas, 2002). Por otra parte, tomando en cuenta el contexto eminentemente relacional del aula, resulta pertinente hacer el alcance de que una de las emociones observadas -etiquetada como "angustia"- puede ser clasificada como "miedo/ansiedad", es decir, surgida como fruto de un déficit de poder percibido (Kemper, 1978). Lo anterior nos lleva a pensar, por ejemplo, por la demanda emocional que de manera específica produciría la estructura jerárquica propia de la escuela sobre las y los docentes, y en cómo el 

significados emocionales de profesoras durante su trabajo en aula. Revista de Sociología, 36(1), 41-56. https://doi.org/10.5354/0719-529X.2021.64425

grado de verticalidad de cada establecimiento podría influir sobre la experiencia emocional docente. No obstante, las tres categorías construidas dan pie a una reflexión que, en congruencia con los aportes de Barrett (2018), ponen en un primer plano el carácter eminentemente subjetivo de las emociones, una consideración que también ha sido enfatizada desde la filosofía con relación al origen y a la manera de aproximarse a los estados mentales. Desde esta disciplina, se plantea que, a diferencia del conocimiento en perspectiva de tercera persona que se utiliza para el estudio de los fenómenos naturales, el acceso a los eventos mentales, -como las emociones-, solo puede realizarse a través de un conocimiento en perspectiva de primera persona (García, 2014). En este sentido, uno de los aspectos más llamativos de los resultados es que, pese a este origen eminentemente subjetivo de las emociones, no todos los significados nucleares estaban orientados internamente, observándose que una parte de éstos apelaban a un origen externo. Es decir, parte de las emociones experimentadas fueron explicadas a partir de razones objetivas (externas) y no subjetivas.

Las categorías elaboradas resultan sugerentes considerando el carácter intersubjetivo (Coll et al., 2008; Wells, 2001), complejo (Doyle, 1986) y sobre todo emocional (Hargreaves, 1998; Zembylas, 2002) del trabajo pedagógico de aula. En dicha labor el o la maestra cumple un rol protagónico (Barber \& Mourshed, 2007), especialmente en la generación de climas emocionales adecuados para el aprendizaje (Casassus, 2008), siendo fundamental la gestión que realice de sus propias emociones (Fernández-Barrocal et al., 2017; Salovey \& Mayer, 1990). Para ello resulta clave si éstas son concebidas por quienes las experimenta como fenómenos de origen subjetivo u objetivo. En caso de lo primero, muy posiblemente las decisiones que se adopten ante su surgimiento apuntarán a gestionar el estado interno y/o su expresión, situación que ha sido descrito a través de conceptos como regulación emocional (Hosotani \& ImaiMatsumura, 2011) o labor emocional (Lindqvist et al., 2019). En cambio, si son concebidas como causadas externamente, es probable que las decisiones tomadas buscarán modificar hechos del ambiente. En dicho caso es razonable suponer un distanciamiento de la responsabilidad personal en la construcción de un clima emocional de aula, lo cual podría entorpecer la consecución de un ambiente propicio para el aprendizaje. En este sentido, cabe destacar la cercanía de este análisis con el concepto de locus de control externo y externo (Rotter, 1966).

La adecuada articulación que un maestro o maestra pueda lograr en el transcurso de una emoción entre lo que ocurre afuera y adentro suyo posiblemente caracterice un buen nivel de consciencia de dicha experiencia. A su vez, una articulación de ese tipo sería reflejo del potencial holístico propio del conocimiento emocional requerido en el trabajo de aula (Zembylas, 2001, 2007). Asimismo, la ausencia de esta articulación y la preeminencia de explicaciones que apuntan a factores externos posiblemente indiquen un nivel discreto de consciencia emocional. Obviamente tales supuestos debieran ser sometidos a verificación en estudios futuros.

Por otra parte, resulta plausible suponer que la destreza para articular adecuadamente los aspectos internos e internos (orientación mixta) de la 
experiencia emocional reflejaría una superación de la noción objetivista de la mente y del mundo social que ha predominado durante el modernismo, y que ha sido cediendo terreno a las posiciones construccionistas. Sin embargo, es necesario relevar que estas últimas aproximaciones no han logrado permear masivamente las prácticas pedagógicas pese a su predominio en el mundo educativo (Pozo et al., 2006), algo que posiblemente se explica debido a que dicho predominio ocurre en el ámbito conceptual (teórico) y no experiencial (fenomenológico) y corpóreo, que es justamente lo que caracteriza al conocimiento proveniente del afecto y la emoción. Por tanto, el fortalecimiento de la consciencia emocional en profesores permitiría avanzar en una comprensión construccionista del trabajado educativo.

Concluyendo, las categorías construidas podrían contribuir a la investigación realizada en este ámbito, enriqueciendo la caracterización de la experiencia emocional en profesores, considerando sobre todo que ésta suele realizarse solo en base a palabras emocionales (Saric, 2015). Además, podría aportar a la exploración sobre el nivel de consciencia con que estos estados mentales son experimentados, cuestión que constituye un aspecto medular de este campo de interés y que en, general, ha sido abordado a través de instrumentos psicométricos que también utilizan solo palabras emocionales. Para lograr lo señalado sería conveniente desarrollar dispositivos que permitan indagar de manera específica sobre las explicaciones que las y los docentes manejan sobre el surgimiento de sus emociones. Dado que la entrevista utilizada fue diseñada para abordar distintos aspectos de la experiencia emocional es posible que su aplicación no agotara la indagación sobre los significados nucleares. Si así hubiera ocurrido es probable que se hubieran construido más de este tipo de significados por participante lo cual, en definitiva, habría permitido despejar con mayor precisión el tipo de orientación que predominó en cada profesora. Por otra parte, si bien la cantidad de participantes posibilitó profundizar en la experiencia emocional de cada una de ellas, contar con una mayor cantidad de profesoras habría permitido explorar una mayor variedad de episodios y significados, más aún si hubieran sido de establecimientos diferentes. No obstante, tal alternativa exige contar con una mayor cantidad de recursos. Evidentemente, la combinación entre cantidad, variedad y profundidad debe ser considerada en investigaciones futuras. Por último, la ausencia de profesores hombres entrevistados constituye sin duda una limitación dadas las diferencias de género que se han observado en esta materia (Demetriou et al., 2009). 


\section{REFERENCIAS}

Aguado, L. (2005). Emoción, afecto y motivación. Alianza.

Albornoz, N., Silva, N., \& López, M. (2015). Escuchando a los niños: Significados sobre aprendizaje y participación como ejes centrales de los procesos de inclusión educativa en un estudio en escuelas públicas en Chile. Estudios Pedagógicos, 41(Número Especial), 81-96. https://doi.org/10.4067/S071807052015000300006

Arias, J., \& Rodríguez, A. (2019). El proceso de enseñanza desde el prisma de las emociones de los docentes. Praxis \& Saber, 10(24), 193-215. https://doi.org/10.19053/22160159.v10.n25.2 019.94

Barber, M., \& Mourshed, M. (2007). How the world's best-performing school systems come out on top. McKinsey and Company.

Barrett, L., \& Russell, J. (2015). The psychological construction of emotion. The Guilford Press.

Barrett, L. (2018). La vida secreta del cerebro. Cómo se construyen las emociones (G. Sánchez Baberán, Trad.). Paidós.

Belli, S., \& Íñiguez-Rueda, L. (2008). El estudio psicosocial de las emociones: Una revisión y discusión de la investigación actual. Psico, 39(2), 139-151.

https://ddd.uab.cat/record/145160

Bericat, E. (2000). La sociología de la emoción y la emoción en la sociología. Papers, Revista de Sociología, 62, 145-176.

https://papers.uab.cat/article/view/v62-bericat
Boiger, M., Ceulemans, E., De Leersnyder, J., Uchida, Y., Norasakkunkit, V., \& Mesquita, B., (2018). Beyond essentialism: Cultural differences in emotions revisited. Emotion, 18(8), 1142-1162.

https://doi.org/10.1037/emo0000390

Brackett, M., Floman, J., Ashton-James, C., Cherkasskiy, L., \& Salovey, P. (2013). The influence of teacher emotion on grading practices: A preliminary look at the evaluation of student writing. Teachers and Teaching: theory and practice, 19(6), 634-646. https://doi.org/10.1080/13540602.2013.827453

Bruner, J. (1986). Realidad mental y mundos posibles. Los actos de la imaginación que dan sentido a la experiencia (B. López, Trad.). Gedisa.

Bruner, J. (1991). Actos de significados: Más allá de la revolución cognitiva (J. C. Gómez \& J. Linaza, Trads.). Alianza.

Cáceres, P. (2003). Análisis cualitativo de contenido: Una alternativa metodológica alcanzable. Psicoperspectivas, 2(1), 53-82.

https://www.psicoperspectivas.cl/index.php/ psicoperspectivas/article/view/3

Casassus, J. (2008). Aprendizaje, emociones y clima de aula. Paulo Freire. Revista de Pedagogía Crítica, 7(6), 81-95.

https://doi.org/10.25074/07195532.6.480

Chen, J., Lee, J., \& Dong, J. (2020). Emotional trajectory at different career stages: Two excellent teachers' stories. Frontiers in Psychology, 11, Artículo 1034.

https://doi.org/10.3389/fpsyg.2020.01034 
Coll, C., Palacios, J., \& Marchesi, A. (Comps). (2008). Desarrollo psicológico y educación. 2. Psicología de la educación escolar. Alianza.

Damasio, A. (1999). El error de Descartes, la razón de las emociones (P. Jaccomet, Trad.). Andrés Bello.

De Sixte, R., \& Sánchez, E. (2012). Cognición, motivación y emoción en la interacción profesor-alumno. Una propuesta para analizar su relación mediante el registro de las ayudas frías y cálidas. Journal for the Study of Education and Development, 35(4),483-496. https://doi.org/10.1174/021037012803495258

Demetriou, H., Wilson, E., \& Winterbottom, M. (2009). The role of emotion in teaching: Are there differences between male and female newly qualified teachers' approaches to teaching? Educational Studies, 35(4), 449-473. https://doi.org/10.1080/03055690902876552

Doyle, W. (1986). Classroom organization and management. En M. C. Wittrock (Ed.), Handbook of research on teaching (pp. 392-431). Mac Millan.

Ekman, P., \& Oster, H. (1979). Facial expressions of emotion. Annual Review of Psychology, 30, 527-554. https://doi.org/10.1146/ annurev.ps.30.020179.002523

Fernández-Barrocal, P., Cabello, R., \& GutiérrezCobo, M. J. (2017). Avances en la investigación sobre competencias emocionales en educación. Revista Interuniversitaria de Formación del Profesorado, 31(1) 15-25.

https://www.redalyc.org/journal/274/274501 36003/html/
Flick, U. (2012). Introducción a la investigación cualitativa (J. Torres Santomé, Trad.). Morata.

Frenzel, A. (2014). Teacher emotions. En R. Pekrun \& L. Linnenbrik-Garcia (Eds.), International handbook of emotions education (pp. 494-519). Routledge.

Fried, L., Mansfield, C., \& Dobozy, E. (2015). Teacher emotion research: Introducing a conceptual model to guide future research. Issues in Educational Research, 25(4), 415-441. https://ro.ecu.edu.au/ecuworkspost2013/2484

Funes, S. (2016). Las emociones en el profesorado: El afecto y el enfado como recursos para el disciplinamiento. Educação e Pesquisa, 43(3), 785-798. https://doi.org/10.1590/S1517-9702201610149719

García, P. (2014). La perspectiva de primera persona como metodología de estudio en filosofía de la mente desde John R. Searle. Revista de Investigación, 5(5), 29-46. https://bit.ly/Garcia2014

Gendron, M., Roberson, D., van der Vyver, J. M., \& Barrett, L. F. (2014). Cultural relativity in perceiving emotion from vocalizations. Psychological Science, 25(4), 911-920. https://doi.org/10.1177/0956797613517239

Hargreaves, A. (1998). The emotional practice of teaching. Teaching and Teacher Education, 14(8), 835-854. https://doi.org/10.1016/S0742051X(98)00025-0

Hernández, R., Fernández, C., \& Baptista, P. (2014). Metodología de la investigación. Mc Graw-Hill. 
Hyry-Beihammer, E. K., Jokikokko, K., \& Uitto, M. (2019). Emotions involved in encountering classroom diversity: Beginning teachers' stories. British Educational Research Journal, 45(6), 1124-1139. https://doi.org/10.1002/berj.3554

Hochschild, A. (2008). La mercantilización de la vida íntima. Apuntes de la casa y el trabajo. Katz Editores.

Hochschild, A. (1975). The sociology of feeling and emotion: Selected possibilities. En M. Millman \& R. M. Kanter (Eds.), Another voice. Feminist perspectives on social life and social science (pp. 280-307). Anchor Books.

Hosotani, R., \& Imai-Matsumura, K. (2011). Emotional experience, expression, and regulation of high-quality Japanese elementary school teachers. Teaching and Teacher Education, 27(6), 1039-1048.

https://doi.org/10.1016/j.tate.2011.03.010

Kadesjö, B., Janols, L., Korkman, M., Mickelsson, K., Strand, G., Trillingsgaard, A. \& Gillberg, C. (2004). The FTF (Five to fifteen): The development of a parent questionnaire for the assessment of ADHD and comorbid conditions. European Child Adolescent Psychiatry, 13, 3-13. https://doi.org/10.1007/s00787-004-3002-2

Keller, M., Frenzel, A., Goetz, T., Pekrun, R., \& Hershey, L. (2014). Exploring teacher emotions: A literature review and an experience sampling study. En P. W. Richardson, S. A. Karabenick, \& H. M. G. Watt (Eds.), Teacher motivation: Theory and practice (pp. 69-82). Routledge.
Khajavy, G. H., MacIntyre, P. D., \& Barabadi, E. (2018). Role of the emotions and classroom environment in willingness to communicate: Applying doubly latent multilevel analysis in second language acquisition research. Studies in Second Language Acquisition, 40(3), 605-624. https://doi.org/10.1017/S0272263117000304

Kemper, T. (1978). A social interactional theory of emotions. John Willey \& Sons.

Lara, A., \& Enciso, G. (2013). El giro afectivo. Athenea Digital. Revista de pensamiento e investigación social, 13(3), 101-119. https://doi.org/10.5565/rev/athenead/v13n3.1060

Le Doux, J. (1998). The emotional brain. Touchtone. Lim, N. (2016). Cultural differences in emotion: Differences in emotional arousal level between the East and the West. Integrative Medicine Research, 5(2), 105-109.

https://doi.org/10.1016/j.imr.2016.03.004

Lindqvist, H., Weurlander, M., Wernerson, A., \& Thornberg, R. (2019). Boundaries as a coping strategy: Emotional labour and relationship maintenance in distressing teacher education situations. European Journal of Teacher Education, 42(5), 634-649. http://doi.org/10.1080/02619768.2019.1652904

Mascolo, M., Fischer, K., \& Li, J. (2003). Dynamic development of component systems of emotions: Pride, shame, and guilt in China and the United States. En R. J. Davidson, K. Scherer, \& H. H. Goldsmith (Eds.), Handbook of affective science (pp. 375-408). Oxford University Press. 
Matson, J., Rotatori, A., \& Helsel, W. (1983). Development of a ranting scale to measure social skills in children: The Matson evaluation of social skills with youngsters (MESSY). Behaviour Research Therapy, 21(4), 335-340. https://doi.org/10.1016/00057967(83)90001-3

Naranjo, C. (2007). Cambiar la educación para cambiar el mundo. La Llave.

Nussbaum, M. (2008). Paisajes del pensamiento. La inteligencia de las emociones. Paidós.

Pekrun, R., \& Linnenbrik-Garcia, L. (2014). International handbook of emotions education. Routledge.

Pekrun, R., Frenzel, A., Goetz, T., \& Perry, X. (2007). The control-value theory of achievement emotions: An integrative approach to emotions in education. En P. Schutz \& R. Pekrun (Eds.), Emotion in education (pp. 13-36). Academic Press.

Petitmengin, C. (2017). Enaction as a lived experience: Towards a radical neurophenomenology. Constructivist Foundations, 12(2), 139-147. http://constructivist.info/12/2/139

Phelps, E., \& Sharot, T. (2008). How (and why) emotion enhances the subjective sense of recollection. Current Directions in Psychological Science, 172), 147-152.

http://doi.org/10.1111/j.1467-8721.2008.00565.x

Pozo, J., Sheuer, N., Pérez, M., Mateos, M., Martín, E., \& de la Cruz, M. (2006). Nuevas formas de pensar la enseñanza y el aprendizaje. Las concepciones de profesores y alumnos. Graó.
Rodríguez, M. (2015). Representación social de la noción de felicidad en estudiantes y profesionales de educación y salud de Chile e Italia. Universitas Psychologica, 14(1), 271-286. https://doi.org/10.11144/Javeriana.upsy141.rsnf

Rodríguez-Corrales, J., Cabello, R., Gutiérrez-Cobo, M., \& Fernández-Berrocal, P. (2017). La influencia de las emociones del profesorado no universitario en la evaluación del rendimiento del alumnado. Revista Interuniversitaria de Formación del Profesorado, 31(1), 91-106. https://www.redalyc.org/journal/274/274501 36014/html/

Romero, S., Rubio-Quintanilla, F., Baser, M., Serrano, R., \& Pastor, M. (2015). Influencia cultural y universalidad en la expresión de las emociones. Una revisión sistemática de estudios recientes. Ágora de Salut, 1, 97-112 http://doi.org/10.6035/AgoraSalut.2015.1.7

Rosselló, J., \& Revert, X. (2008). Modelos teóricos en el estudio científico de la emoción. En F. Palmero \& F. Martínez (Eds.), Motivación y emoción (pp. 95-137). McGraw-Hill.

Rotter, J. (1966). Generalized expectancies for internal versus external control of reinforcement. Psychological Monographs: General and Applied, 80(1), 1-28.

https://doi.org/10.1037/h0092976

Salovey, P., \& Mayer, J. (1990). Emotional intelligence. Imagination, Cognition, and Personality, 9(3), 185-211. https://doi.org/10.2190/DUGGP24E-52WK-6CDG 
Saric, M. (2015). Teachers' emotions: A research review from a psychological perspective. Journal of Contemporary Educational Studies, 4, 10-26. https://bit.ly/Saric2015

Solana, M., \& Vacarezza, N. (2020). Sentimientos feministas. Revista Estudos Feministas, 28(2), Artículo e72445. https://doi.org/ 10.1590/1806-9584-2020v28n272445

Tsang, K. (2014). A review of current sociological research on teacher's emotions: The way forward. British Journal of Education, Society \& Behavioural Science, 4(2), 241256. https://ssrn.com/abstract=2362149

Timoštšuk, I., Kikas, E., \& Normak, M. (2016). Student teachers' emotional teaching experiences in relation to different teaching methods. Educational Studies, 42(3), 269-286. http:/doi.org/10.1080/03055698.2016.1167674

Uitto, M., Jokikkoko, K., \& Estola, E. (2015). Virtual special issue on teachers and emotions in Teaching and teacher education (TATE) in 1985-2014. Teaching and Teacher Education, 50, 124-135.

https://doi.org/10.1016/j.tate.2015.05.008

Varela, F., Thompson, E., \& Rosch, E. (1997). De cuerpo presente. Las ciencias cognitivas y la experiencia humana (2da ed.). Gedisa.

$\mathrm{Xu}, \mathrm{Y}$. (2018). A methodological review of L2 teacher emotion research: Advances, challenges and future directions. En J. de D. Martínez (Ed.), Emotions in second language teaching: Theory, research and teacher education (pp. 35-49). Springer. https://doi.org/10.1007/ 978-3-319-75438-3_3
Zembylas, M. (2001). A paralogical affirmation of emotion's discourse in science teaching. En M. Osborne \& A. Barton (Eds.), Teaching science in diverse settngs: Marginalized discourses and classroom practice (pp. 99-128). Peter Lang.

Zembylas, M. (2002). Constructing genealogies of teachers' emotions in science teaching. Journal of Research in Science Teaching, 39(1), 79-103. https://doi.org/10.1002/tea.10010

Zembylas, M. (2007). Emotional ecology: The intersection of emotional knowledge and pedagogical content knowledge in teaching. Teaching and Teacher Education, 23(4), 355367. http://doi.org/10.1016/j.tate.2006.12.002

Zembylas, M., \& Schutz, P. (Eds.). (2016). Methodological advances in research on Emotion and education. Springer.

Zenteno-Osorio, S., \& Leal-Soto, F. (2016). Los afectos en la experiencia de ser profesor de un estudiante diagnosticado con trastorno espectro autista. Pensamiento Educativo. Revista de Investigación Educacional Latinoamericana, 53(1), 1-14. https://doi.org/10.7764/PEL.53.1.2016.5

Manuscrito recibido: 27-02-2021 Manuscrito aceptado: 13-07-2021 